\title{
Energy-Delay Efficient Unified Routing Protocol for Wireless Sensor Networks
}

\author{
V. P. Jayachitra ${ }^{*}$, G. Geetha ${ }^{2}$, K. Santhan Vijay ${ }^{1}$ \\ ${ }^{1}$ Department of Computer Technology, MIT Campus, Anna University, Chennai, India \\ ${ }^{2}$ Department of Computer Science \& Engineering, Jerusalem College of Engineering, \\ Anna University, Chennai, India \\ Email: ${ }^{*}$ jayachitravp@annauniv.edu, togeethamohan@gmail.com
}

Received 17 March 2016; accepted 21 May 2016; published 24 May 2016

Copyright (C 2016 by authors and Scientific Research Publishing Inc.

This work is licensed under the Creative Commons Attribution International License (CC BY). http://creativecommons.org/licenses/by/4.0/

(c) () Open Access

\begin{abstract}
One of the most important challenges in the Wireless Sensor Networks is to improve the performance of the network by extending the lifetime of the sensor nodes. So the focus is on obtaining a trade-off between minimizing the delay involved and reducing the energy consumption of the sensor nodes which directly translate to an extended lifetime of the sensor nodes. An effective Sleep-wake scheduling mechanism can prolong the lifetime of the sensors by eliminating idle power listening, which could result in substantial delays. To counter this, an anycast forwarding scheme that could forward the packet opportunistically to the first awaken node may result in retransmissions as if the chosen node falls in resource constraints. The algorithm, namely Prim'sDual is proposed to solve the said problem. The algorithm considers five crucial parameters, namely the residual energy of the nodes, transmission power, receiving power, packet loss rate, interference from which the next hop is determined to extend the lifetime of the sensor node. Since the proposed work is framed keeping critical event monitoring in mind, the sleep-wake scheduling is modified as low-power, high-power scheduling where all nodes are in low-power and the nodes needed for data transmission are respectively turned on to high-power mode. The integrated framework provides several opportunities for performance enhancement for conflict-free transmissions. The aim of our algorithm is to show reliable, energy efficient transfer without compromising on lifetime and delay. The further effectiveness of the protocol is verified. The results demonstrate that the proposed protocol can efficiently handle network scalability with acceptable latency and overhead.
\end{abstract}

\section{Keywords}

Angle Based Routing, Cross-Layer, Energy-Delay Trade-Off, Medium Access Control (MAC), Prims-Dual Minimum Spanning Tree

\footnotetext{
${ }^{*}$ Corresponding author.
} 


\section{Introduction}

Recent broad spectrum utilization of Wireless Sensor networks has led to this domain being a very active and rich field of research. In mission-critical applications, such as battlefield reconnaissance, forest fire detection, and gas monitoring requires fast response time and the traffic distribution in such applications are often bounty [1]. Also, the communication environment is harsh because of multipath effects, high path loss and Co-channel interference, leading to high energy consumption and maximizes the delay [2]. Additionally, WSNs (Wireless Sensor Networks) are usually constrained by the low-cost and low-power consumption requirements for the sensor components, enforcing the communication technology to be light-weight and low-power i.e. IEEE 802.15.4 [3]. Added, replacing or recharging the sensor's battery is impossible in a harsh environment. Due to such hard energy constraints, efficient use of energy is one of the main design considerations in WSNs. On the other hand, some critical applications require fast response time that enforces some constraints on the source to sink delay. Thus, finding a trade-off between energy consumption and delay is very critical since, satisfying either one of them will not satisfy the other [4].

Traditionally, protocol architectures follow strict layering principles, which limit the coordination among the layers limits the performance of wireless nature of the transmission links. To overcome such limitations, crosslayer design to enhance the wireless network performance such as energy efficiency, increase network capacity and improves QoS (Quality of Service) of the overall system had been introduced [5] [6]. The proposed paper EDURo $^{+}$allows physical, MAC and network layers to coordinate and interact crossing different layers to attain trade-off between QoS.

Most of the energy is consumed when the nodes are active, listening for an event to occur. At MAC layer level, sleep-wake scheduling connected-k neighborhood (CKN) [7], geographic-distance-based connected-k neighborhood GCKN [8] was proposed as an effective mechanism in which the node is active briefly and sleeps for a long time to prolong the network lifetime. Though in sleep-wake schedule, the sender node has to wait until the neighbor node wake up and hence incur delays in transmission. In sleep window MAC (SW-MAC) [9], the wake-up rate can be tuned according to the current traffic of the network which exhibits collision due to synchronization and is not reliable. However, asynchronous MAC scheduling can awake or sleep by transmitting a preamble longer than receivers wake up rate without the constraint of synchronization mechanism. But too long preamble, Berkeley MAC (B-MAC) [10] increases the delay and too short preamble, X-MAC (Extended MAC) [11] might increase energy. The B-MAC and X-MAC based MAC (BoX-MAC-1) [12] protocol uses data packet as probe packet instead of preambles and suited for sparse networks as the energy and collision increase linearly with node density. A state-of-the-art receiver initiated MAC protocol, A-MAC [13] performs poorly in networks with asymmetric links degrading Packet Reception Ratio [PRR] as the sender node fails to receive a probing packet from the anticipated receiver. The most recent work that addressed the above problem was Asym-MAC [14], a MAC protocol designed for low-power duty-cycled WSNs with asymmetric links. More specifically, for asymmetric link, the transmitter-initiated and default MAC for receiver-initiated is used. The hybrid method achieves up to $66.7 \%$ lesser delay compared to A-MAC protocol.

However, in the case of critical event monitoring the above MAC scheduling algorithms do not fit well. So an efficient algorithm with shorter preamble and faster selection for switching the mode to the high-power mode and packet transfer is needed.

At the Network layer level, the design of low complexity energy efficient routing protocol such as Geographic routing, multi-hop routing, opportunistic routing and anycast routing provides a method to reduce the energy cost of the network. A Geographic energy efficient routing (EER) [6] balances only energy consumption in networks. Similarly, many multihop routing algorithms Ad hoc On-Demand Distance Vector Routing (AODV), Destination-Sequenced Distance Vector routing(DSDV) and Dijkstra's algorithm balances energy consumption by finding efficient routing path without considering a delay. However, Delay can be controlled by implementing the concept of tree based path routing. There are many WSN algorithms focus on particular parameters like Energy-efficient Opportunistic Routing Technology (EFFORT) [15] or interference-aware routing (LAR) [16], whereas in Energy-Efficient Unified Routing (EURo) [17] a combination of four parameters viz, transmission power, interference, residual energy, and energy replenishment was considered. It uses an existing shortest path algorithm to find the minimum cost route and incurs a high delay.

Anycast forwarding scheme is a network addressing and routing methodology in which datagram's from a single sender can be forwarded to the first node that wakes up in the forwarding set. This mechanism of for- 
warding datagram's to any member of its forwarding set reduces sleep latency. Each of them focuses on reducing the transmission latency and thereby balances the energy of the network [18]-[22]. The selection of the forwarding scheme in anycast (normal) is based on distance, anycast (naïve) is based on communication range, anycast (optimal) [23] chooses the forwarder set optimally and deterministic [24] chooses one forwarder node based routing were proposed. A new novel anycast algorithm known as Geographic K-anycast Routing (GKAR) [25] was framed to find paths from source node $S$ to at least $K$ destinations out of all the destinations in $D$, under the constraint that some destinations in D may be unavailable with S. Note that if less than $\mathrm{K}$ destinations are available in $\mathrm{S}$, no routing protocol can reach $\mathrm{K}$ destinations. Besides, guaranteeing to reach $\mathrm{K}$ destinations eventually, the energy consumption should also be minimized. Although the above anycast routing methodology addresses the efficient routing mechanism by considering only energy and delay factors, but there is no coordination between sender and receiver regarding resource information.

An opportunistic based energy efficient routing strategy which prioritizes a forwarder set to forward data to the sink node in WSNs was introduced in Energy efficient opportunistic routing (EEOR) protocol [26]. In ASSORT (asynchronous sleep-wake scheduling and opportunistic routing) [27] and in D-SW (Deterministic routing and sleep-wake scheduling) [28] were considered to enhance the network lifetime. However, in the case of critical event monitoring applications, sleep-wake schedule is not advisable to use. The recent work included an energy-efficient routing algorithm for 1-D queue network, namely, Energy Saving via Opportunistic Routing (ENS_OR) [29]. ENS_OR selects and prioritize a forwarder set according to their virtual optimal transmission distance and residual energy level. Moreover balancing between energy and delay was not guaranteed. While all of these routing methods minimize energy consumption of individual node or the whole network, it is equally important to focus on other metrics such as transmission \& receiving power, interference, distance between the sender and receiver, and residual energy of both sender and receiver along with vigilant scheduling is needed.

In this paper, an Energy-Delay Unified Routing $\left(\right.$ EDURo $\left.^{+}\right)$protocol was proposed which unifies the crucial parameters like Transmission power, Receiving power, Interference, Distance between the two communicating nodes, and Remaining Energy of the nodes. The nodes are currently in the low-power mode and are switched to high-power mode when needed for packet transfer. The EDURo ${ }^{+}$employs a better Minimum Spanning Tree Algorithm called Prim's Dual that is used to determine the next-hop using the available parameters. This approach that considers both the sender and receiver metric is supported by an efficient scheduling algorithm that focuses on reducing delay. The packet transfer is supported by anycast routing methodology which uses a geographic location (GPS) to determine the position of the destination in the forward direction. This will guarantee good balancing between energy consumption and delay with less overhead.

The rest of this paper is organized as follows; Notations, Assumption of EDURo ${ }^{+}$defined in Section 2. Section 3 defines our proposed work EDURo ${ }^{+}$in detail. We illustrate the simulation results of our approach that compare the EDURo ${ }^{+}$performance with other existing approaches in Section 4. And finally, we conclude and summarize our contributions in Section 5.

\section{System Model and Notations}

\subsection{Network Model}

We consider a multi-hop WSN with $N$ sensor nodes, which can be modeled by a communication graph $G=(U$, $L$ ), where $U=\left\{u_{1}, u_{2}, \cdots, u_{n}\right\}$ is the set of normal sensor nodes excluding the sender and the receiver node and $L$ is the set of links. The nodes are said to be static with an initial energy of full capacity. The default transmission radius of each sensor is $t_{r}$ and the maximum transmission radius of each sensor is $t_{r m}$. Two sensors are neighbors if they are within the transmission range. A link $l(u, v) \in L$ if nodes $u$ and $v$ can communicate with each other directly without relaying. Two sensors $u$ and $\mathrm{v}$ are two hop neighbors if $l(u, v) \in L$ and there exists another node $w$ satisfying $l(u, w) \in L, l(w, v) \in L$, or $l(v, w) \in L, l(w, u) \in L$.

\subsection{Assumptions and Notations}

We assume that each node knows its own location by using a Global Position System (GPS) receiver or some mobility based localization algorithm. We also assume that all the nodes are in a low-power mode and switched 
to high-power mode when a particular node is selected for data transmission. We further assume that each node also knows the locations of the source and sink nodes by flooding or opportunistic flooding. Specifically, as each sensor knows its own location, if the destination is static, the destination location information only needs to be flooded once. If the sink is mobile and normal sensor nodes are static, the sink location information needs to be flooded when it moves to a new location. The notations used in EDURo ${ }^{+}$are represented in Table 1.

\subsection{System Overview}

We consider the system consist of sensor nodes and an active sink. The sensor nodes are densely deployed in a multi-hop wireless sensor network. The events detected by the sensor node are encapsulated into packets and those packets are forwarded to the only sink using multi-hop forwarding. All the nodes are static and each node is provided with the energy of full capacity initially. Global positioning system (GPS) is used to know the location of each node in the network. The location information of the destination node is flooded once in the network for routing. In this system cross-layer design involves three layers namely, i) physical layer, ii) MAC layer, iii) network layer. These layers include three main functions:

i) Angle based routing

ii) Low-high power vigilant scheduling and

iii) Route selection (Prim's Dual) to provide energy efficient and delay minimized routing.

The data transmission between two nodes occurs only in the high-power mode. The overall system view is depicted in Figure 1.

\section{Protocol Design [EDURo+]}

The Energy-Delay Unified Routing protocol [EDURo ${ }^{+}$] consists of Configuration and Initialization phase. The following section briefs the functionality of each phase with diagram representation.

\subsection{Configuration Phase}

Initially the nodes in the network evaluate the control parameters by following anycast forwarding scheme. The Low power High power scheduling pattern is initiated during this phase and hence sensor nodes will not follow asynchronous sleep-wake mechanism.

Table 1. The notations used in EDURo ${ }^{+}$.

\begin{tabular}{cc}
\hline Symbol & Description \\
\hline$E_{\text {Remaining }}$ & Energy currently available in the node \\
$E_{\text {Initial }}$ & Initial energy of the node \\
$E_{\text {Transmission }}$ & Energy needed by the node for transmission \\
$E_{\text {Receiving }}$ & Energy needs by the node for receiving packet \\
PLr & Packet Loss Rate of the Node \\
$T$ & Source node part of the tree \\
$T$, & V/\{source node not part of tree $\}$ \\
$E_{\mathrm{T}}$ & Edges in the tree \\
$\mathrm{PP}$ & For all $\mathrm{j}$ in V [potential parent] \\
$\mathrm{PC}$ & For all i in V [potential child] \\
$\mathrm{RIT}$ & Receiver Initiated Transmission \\
$\mathrm{NAV}$ & Network Allocation Vector \\
$\mathrm{CTS}$ & Clear To Send \\
$\mathrm{ACK}$ & Acknowledge \\
\hline
\end{tabular}




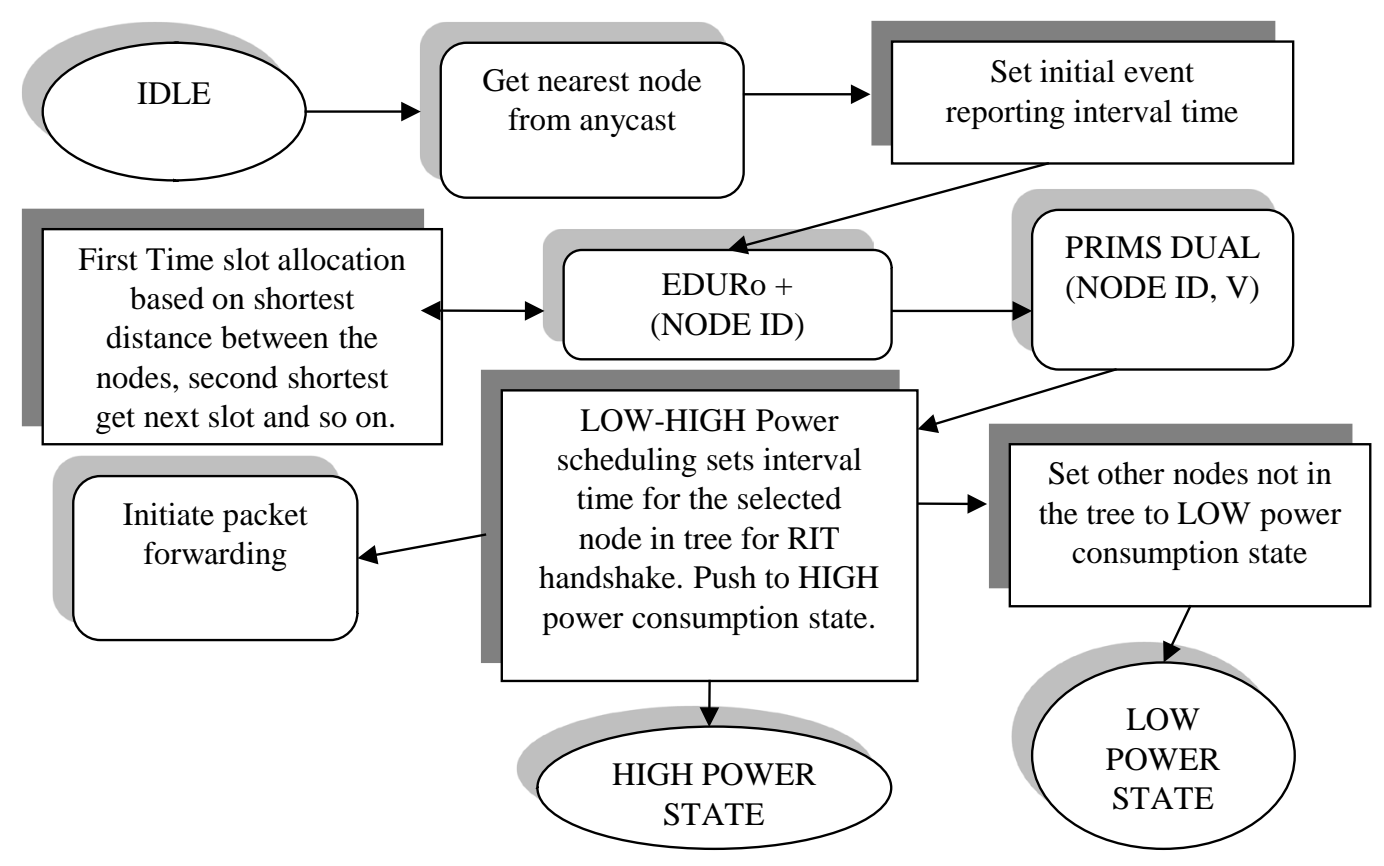

Figure 1. EDURo ${ }^{+}$system overview.

\subsection{Operation Phase}

In operation phase, each node switches between the low power sub-phase and the high power sub-phase. In the low power sub-phase, each node simply waits for an event to occur. Finally, when an event occurs, the angle based forwarding set is determined and applies prim's dual algorithm to determine the routing path, using the control parameters chosen during the configuration phase. In prim's dual, edges in MST are determined by a set of various parameters from three layers of both sender and receiver that are crucial to energy consumption and delay factor which as a whole contribute to lifetime and other important metrics in wireless sensor networks. The nodes that form the vertices in MST switches to high-power and the transmission begins. Figure 2 shows the parameter set considered in the proposed algorithm. Parameters are considered from the network layer, data link layer and the physical layer of the cross-layer framework to determine the next hop.

The modules in the operation phase are

a) Threshold Calculation.

b) Prim's Dual Algorithm [PMD].

c) Low power-High power Scheduling [LHS].

\subsubsection{Threshold Calculation}

For the next hop selection, both the parameters of the sender and the receiver are considered. Since it doesn't sound convincing to collaborate the parameters of both the sender and receiver into a single threshold, we propose two threshold's one that by the sender and one by the receiver. We propose sender based threshold as metric one that considers the energy consumption ratio (remaining energy/ initial energy) and the energy required for transmission as in Equation (1). Next, we propose a receiver based threshold as metric-two that considers the energy consumption ratio (remaining energy/initial energy), the energy required for receiving process and the nodes packet loss rate as in Equation (2). Equations (1) and (2) are two threshold's used by prim's dual to select the next hop.

$$
\begin{gathered}
T(1)=\frac{E_{\text {Remaining }}}{E_{\text {Initial }}} \times E_{\text {Transmission }} \\
T(2)=\frac{E_{\text {Remaining }}}{E_{\text {Initial }}} \times E_{\text {Receiveing }} \times \mathrm{PLr}
\end{gathered}
$$




\subsubsection{Prim's Dual Algorithm [PMD]}

The given communication network is a graph $\mathrm{G}(\mathrm{V}, \mathrm{E})$ with nodes $\mathrm{V}$ and links $\mathrm{E}$. There is a sink node among $\mathrm{V}$ and an edge $\{i, j\}$ has the weight $\mathrm{W}_{\mathrm{ij}}$. Initially, the partial spanning tree only contains the sink. During the execution of the PMD algorithm, new links are added to the partial spanning tree and at the end, a spanning tree is constructed. The basic operations that are executed in the iterations of the Algorithm 1 (PMD) are as follows:
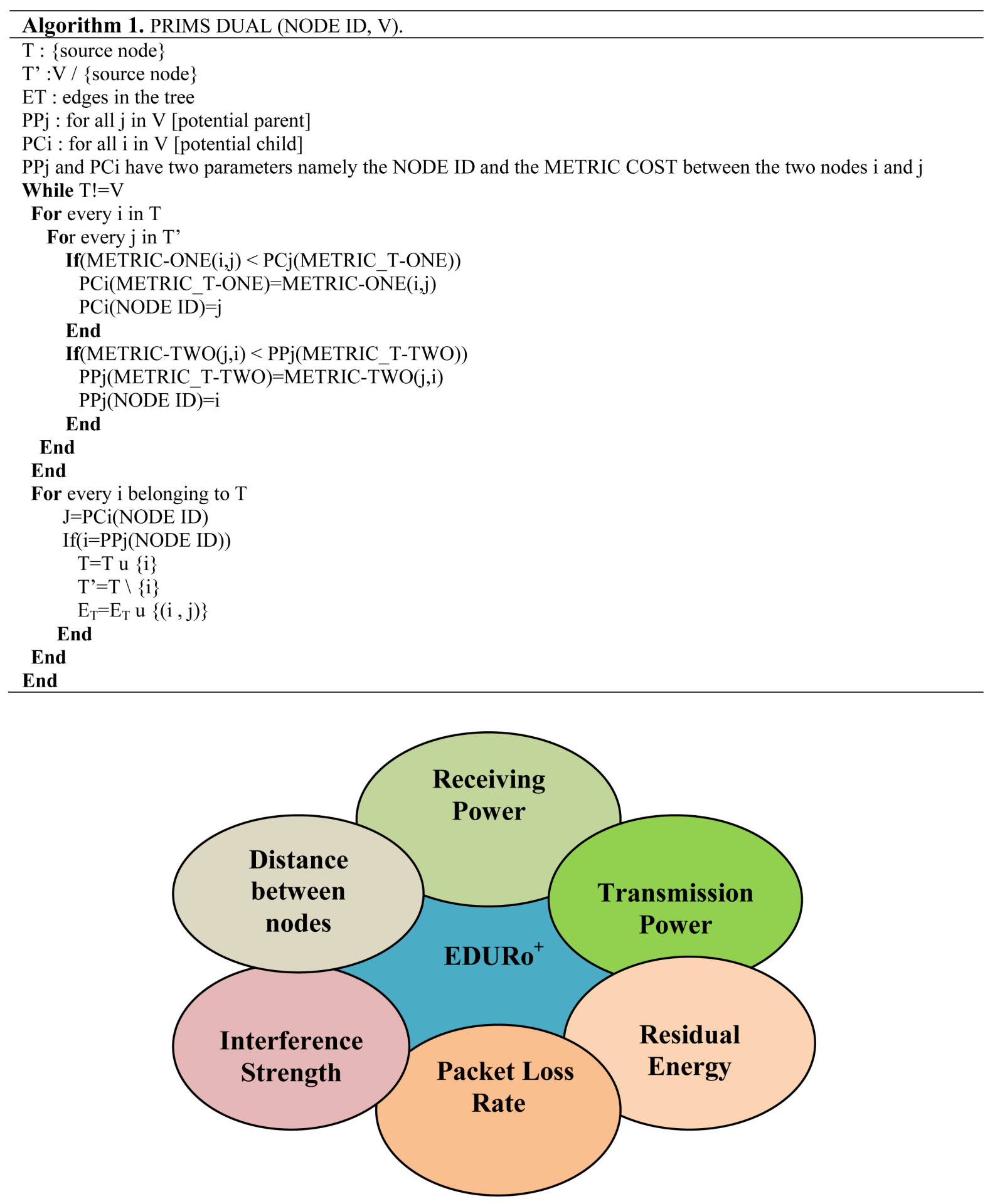

Figure 2. EDURo ${ }^{+}$control parameters. 
1) Each node in the current partial spanning tree selects a potential child node (if any) from the nodes that are not added to the current tree yet.

2) Each node of the graph that is not a member of the current tree selects a potential parent (if any) among the nodes in the current tree.

3) The nodes in the current tree which are the potential parent of their potential child set up a link with these nodes.

The potential nodes are decided by the above two threshold's Metric-one (1) and Metric-two (2). Both PPj and PCi have two parameters namely the NODE ID and the METRIC COST between the two nodes, node $\mathrm{i}$, and node j respectively. The basic steps involved in constructing the Prims Dual minimum spanning tree are explained in the Figure 3.

An illustrative example of a link setup in PMD is shown in Figure 4 and Figure 5. The source node is in red and the blue nodes with numbers 2 - 5 are other nodes existing in the current tree. The solid black lines are the

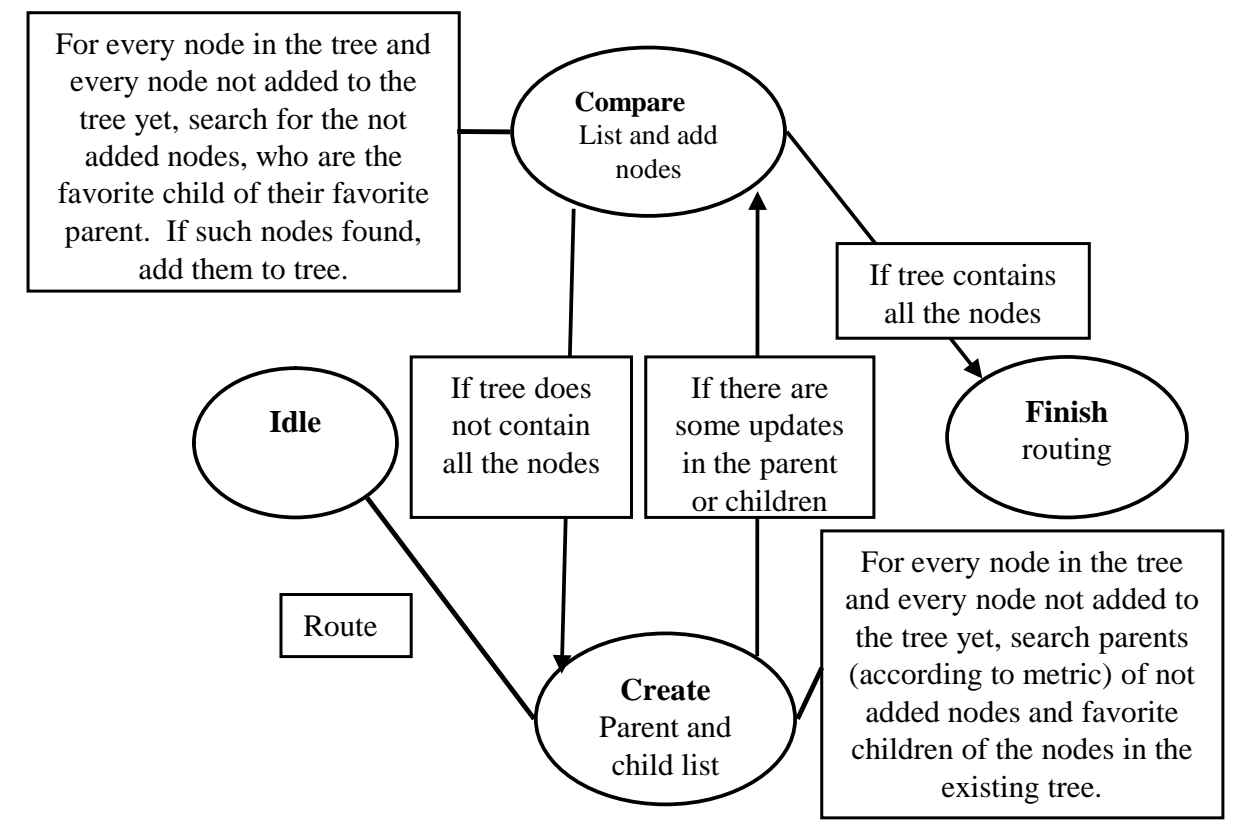

Figure 3. Flow chart of the Prims dual algorithm for constructing MST.

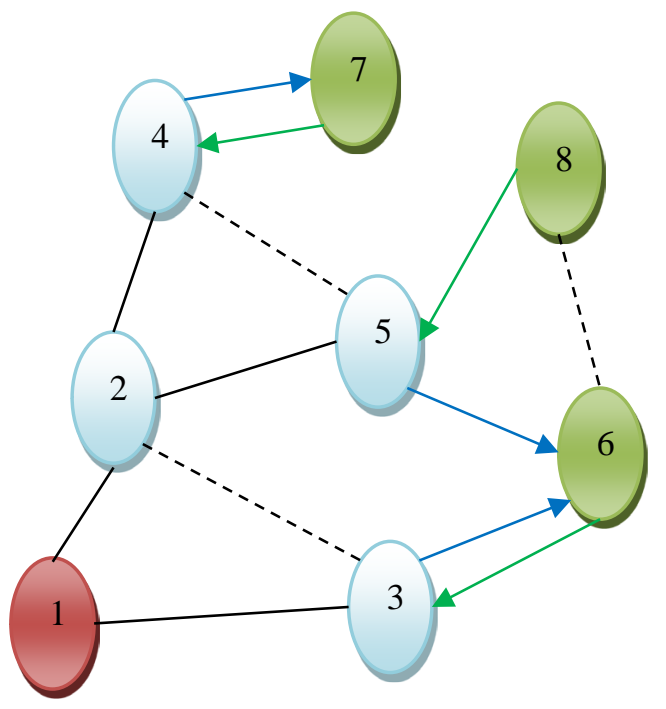

Figure 4. Initial setup. 


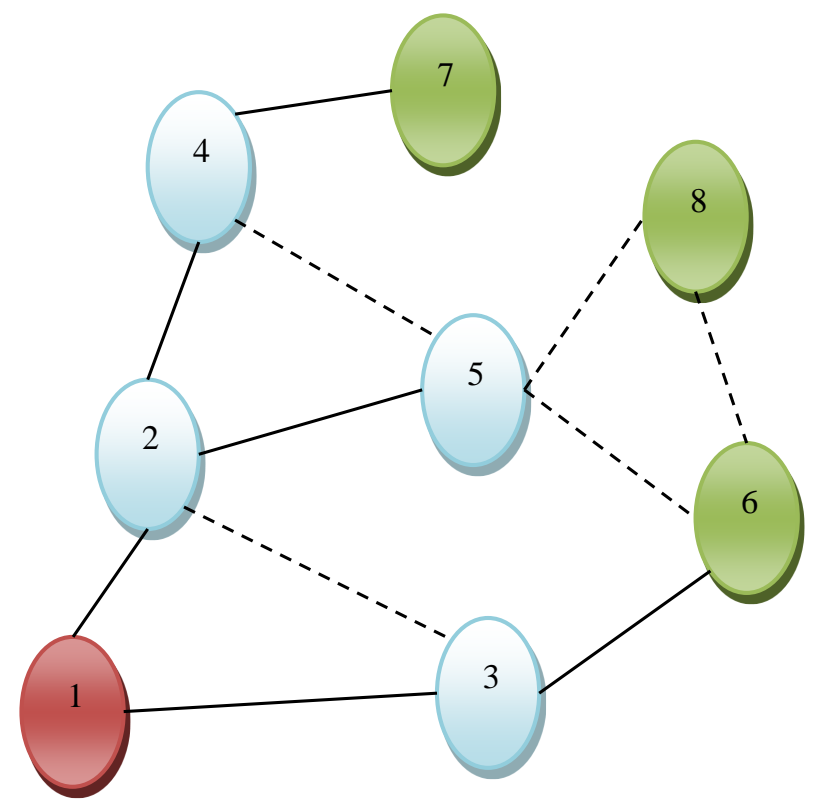

Figure 5. Final setup after Prim’s Dual.

links of the current tree and the dotted black lines are the possible links. The nodes 6,7 and 8 are potential child nodes, which are not added to the tree yet as shown in Figure 4. The node 7 selects node 4 as its potential parent (green arrow), whereas the node 4 selects node 7 as a potential child (blue arrow). The same happens with node 6 and 3 as well. Node 5 selects node 6 as its potential child and node 8 selects node 5 as its potential parent. After links are constructed with one prim's dual algorithm iteration, we obtain the partial spanning tree shown in Figure 5. In Figure 5, Node 6 and Node 7 are connected to the tree, whereas; the node 8 could not be added to the tree because it was not selected by its potential parent as the metrics were not satisfied.

\subsubsection{Low-High Power Scheduling [LHS]}

In the event reporting phase, first, the position of the sender and the receiver node using GPS is received. Then we initiate an angle of $45^{\circ}$ using any cast event reporting methodology in the forward direction from the information received by a GPS. Short preambles or beacons are sent by the sender at short intervals which are shorter than X-MAC since only nearest nodes are returned by any casting methodology. When a node in the sensing region responds, it initiates a CCA (clear channel assessment). When the channel is free it sends a probe packet to the sender. After receiving the probe packet, a minimum spanning tree from the sender is constructed using Prims Dual algorithm by assessing the potential nodes. During data transmission, a receiver which is potential of receiving data, switches to high power and sends a set of two packets RIT (Receiver Initiated Transmission) to the sender and NAV (Network Allocation Vector) to its neighbor (RIT hand-shake).

The whole process of EDURo ${ }^{+}$-LHS can be visualized as a four-way handshake as indicated in Figure 6. During data transmission, a receiver which is potential of receiving data, switches to high power and sends an RIT packet (Receiver Initiated Transmission)to the sender and overheard by a receiver adjacent node is the first step of the four-way handshake (RIT Handshake). Upon receipt of RIT from the receiver, the sender prepares NAV (Network Allocation Vector) packet to be sent to its adjacent nodes having a node ID and time-slot value as the prime information indicating busy channel (NAV Handshake). Data transfer is then initiated from sender to receiver node within the time-slot (DATA transfer Handshake). After the data is received an ACK packet is sent to complete the transaction (ACK Handshake).

In Figure 7, RIT packet is sent from the selected node, i.e. Node 2, to the sender i.e. Node 3, acknowledging that it is ready to receive data, removing the need for RTS. NAV is then sent from Node 3 to its adjacent nodes indicating it is busy in data transfer and that it is set to the time same as that of data transmission time i.e. time slot value, thus avoiding hidden and exposed the terminal problem. This process is repeated till the destination node is reached as indicated in the Algorithm 2. 

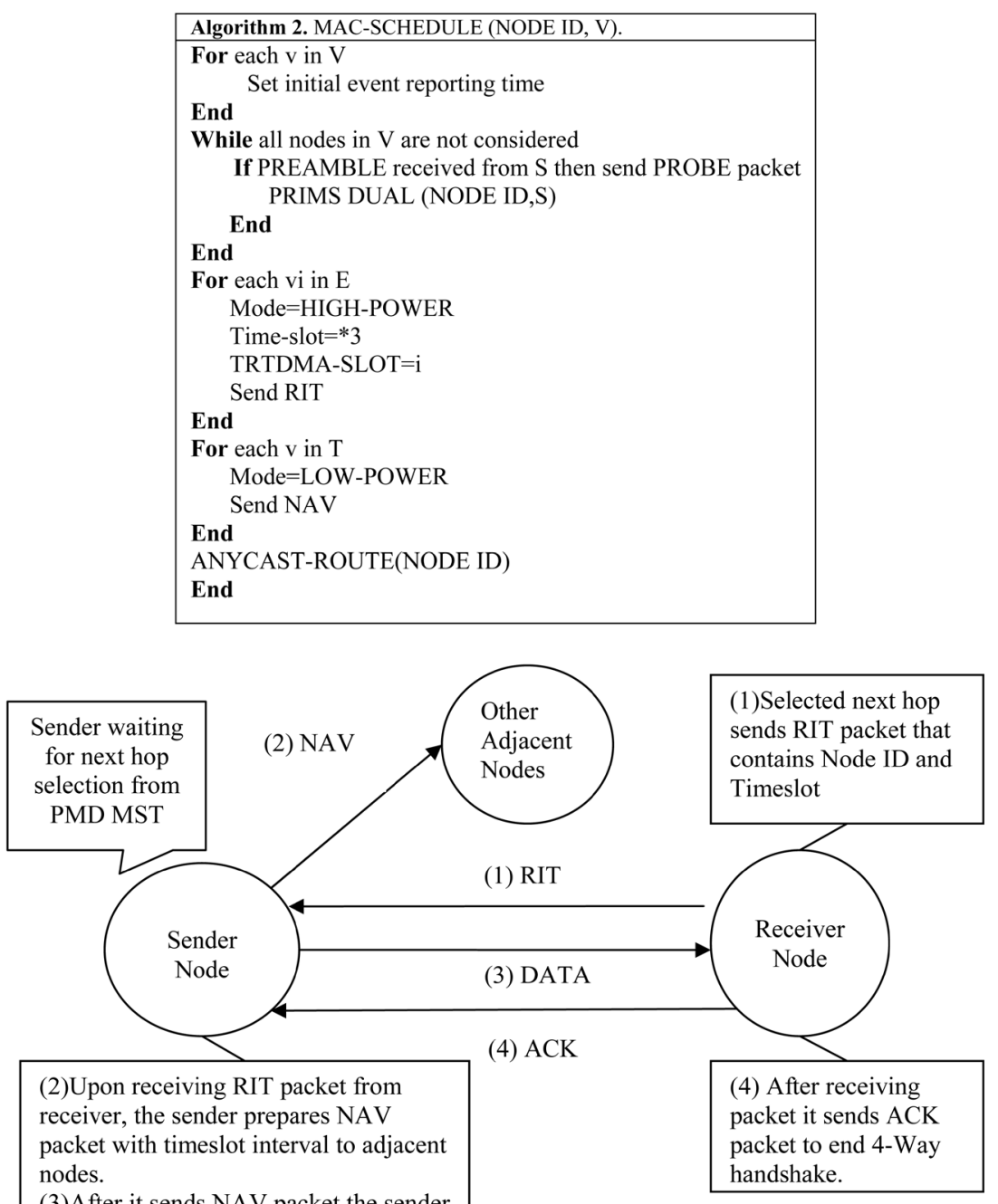

(3)After it sends NAV packet the sender

begins sending data to the receiver node within the allotted time slot.

Figure 6. EDURo ${ }^{+}$-LHS 4-way Handshake mechanism.

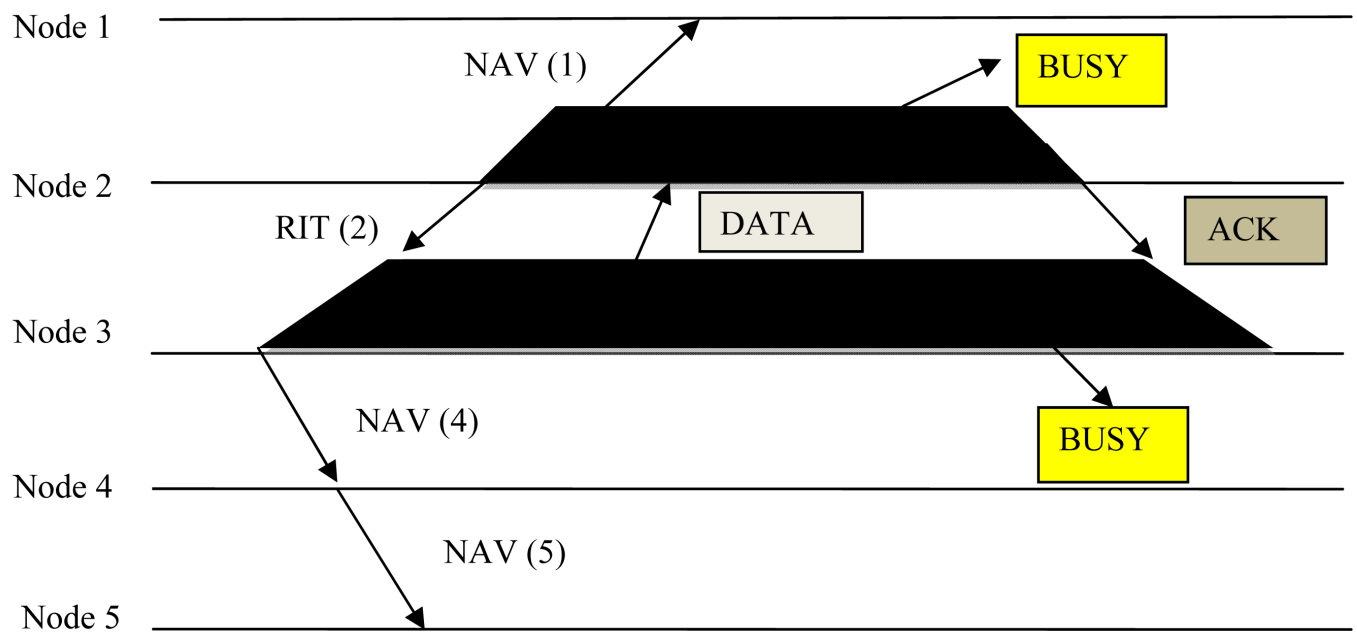

Figure 7. EDURo ${ }^{+}$LHS handshake mechanism. 


\section{Performance Evaluation}

We simulated the proposed work under NS2 Simulator and investigated the performance of similar work. In the simulation setup, we consider a static sink node and static 100 nodes uniformly distributed random way on a 100 $\mathrm{m} \times 100 \mathrm{~m}$ area. We consider the impact of unevenly distributed initial battery energy and the sink node with unlimited energy. We assume that the initial battery energy of the network follows a uniform distribution between 5 and 15 units of energy so that the mean of the distribution is 10 units of energy. Due to the variation of battery energy, the thresholds in the algorithm play a more important role in choosing a route than those in the homogeneous battery case. The performance metrics used in the evaluation of EDURo ${ }^{+}$protocol are Energy and delay. The minimum remaining energy for the proposed work does slightly better than other work as the latter considers very few parameters and also EDURo ${ }^{+}$puts nodes, not for use in low power as indicated in Figure 8.

The total energy consumption of the proposed algorithm was compared with existing ones and is shown in Figure 9. We observe that the energy consumption of the sensors is more balanced in EDURo ${ }^{+}$, ASSORT, ANYCAST, D-SW than in EFFORT. This is because former considers the residual energy of sensor nodes, and the nodes with more remaining energy are selected as forwarders. Also that the total energy consumption in EFFORT is higher than those of ASSORT, ANYCAST, and D-SW by 71\%, 91\%, and 55\%, because sensor nodes in EFFORT never go to sleep mode for energy conservation when they are idle. Moreover, we observe that the total energy consumption in ANYCAST is slightly higher than that of the ASSORT by 11\%. This can be explained as follows. ASSORT does not need to let multiple forwarders wake up simultaneously for data forwarding which allows sensor nodes to sleep longer. EDURo ${ }^{+}$performs much better than ANYCAST but slightly consumes a bit more energy than ASSORT by a mere 3\% - 5\% since it is not asymmetric and it is also receivercentric.

However, the links with poor reliability would spend more energy for retransmissions, which incurs unbalanced energy consumption than EDURo ${ }^{+}$. Besides, because D-SW does not exploit opportunistic routing to reduce the energy consumption caused by unreliable wireless links and hence the total energy consumption of D-SW is lower than that of ASSORT. Figure 10 shows the comparison of the proposed EDURo ${ }^{+}$-LHS with the latest versions of MAC protocols. In comparison with AMAC, the receiver of Asym-MAC consumes more energy which is the cost for Asym-MAC's CCA checks. Similarly, the transmitter of Asym-MAC consumes more energy than A-MAC; however, interestingly, the difference is not large with an increase of only $2.67 \%$, implying that the transmitter of A-MAC consumes energy unnecessarily waiting for the probing packet especially when the link is asymmetric, although Asym-MAC consumes energy by changing the Mode and that transmitting the preambles. Since Eduro ${ }^{+}$-LHS are receiver involved and it consumes a bit more energy when

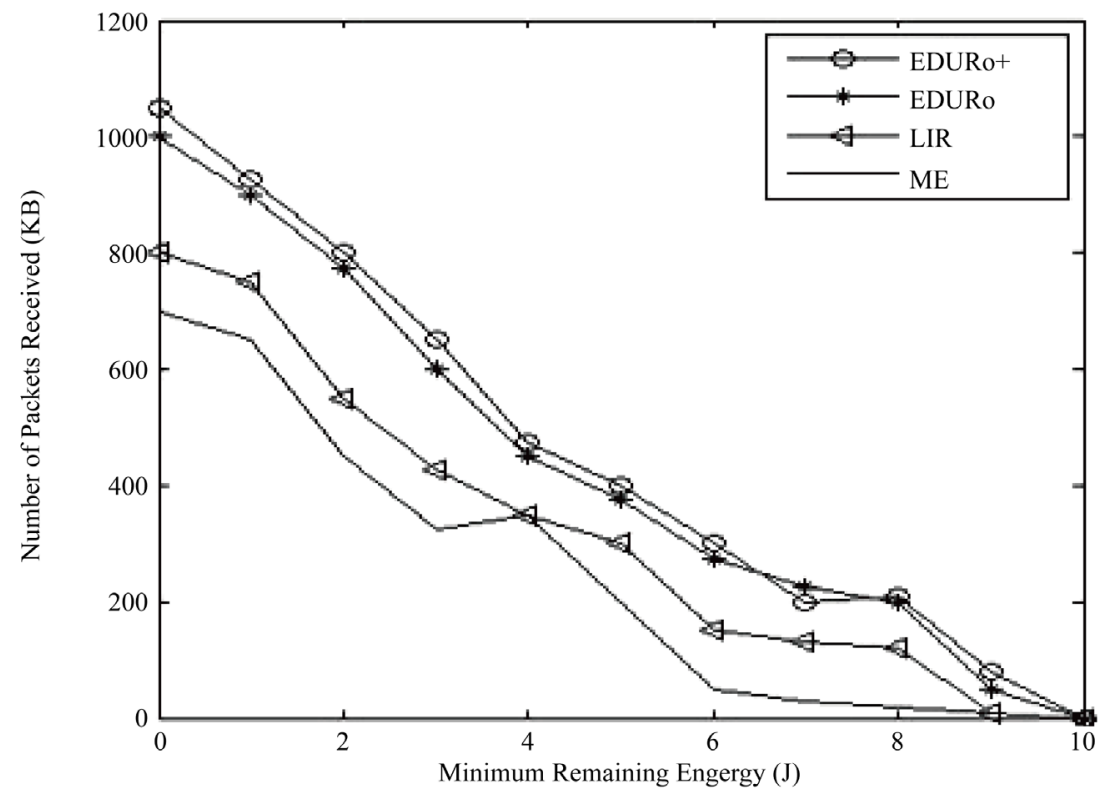

Figure 8 . The minimum remaining energy vs the number of arrived packets. 


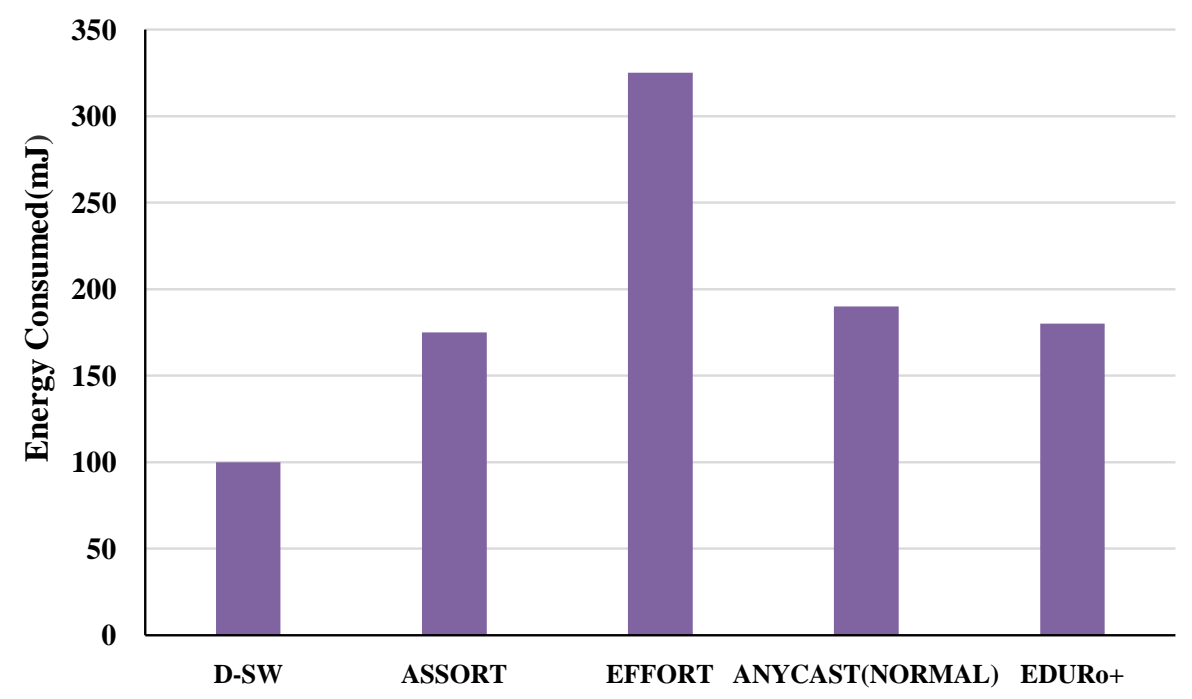

Figure 9. The total energy consumption for 100 nodes.

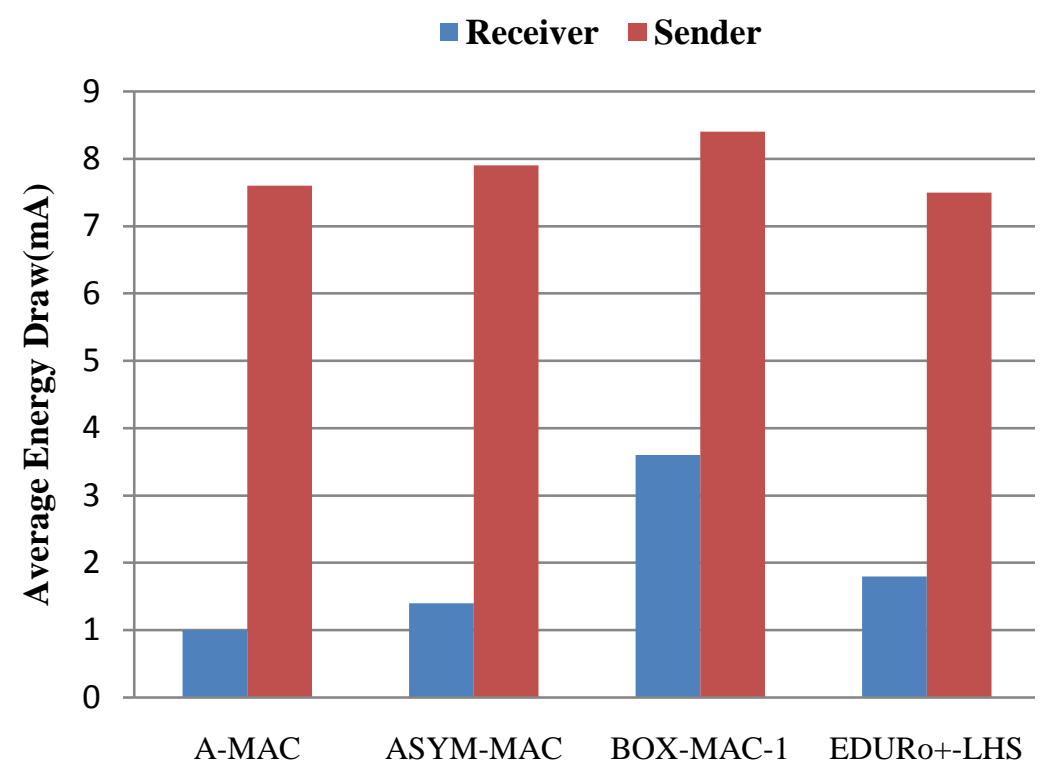

Figure 10. Average energy consumption of MAC protocols.

compared to Asym-MAC but it is correlated by its sender. The proposed LHS scheduling fares better when compared with the receiver initiated protocols, the graph shows BoX-MAC-1 has higher energy consumption, which coincides with the reported results.

In Figure 11, we have a look at the other parameter namely delay. Anycast (optimal) was better in terms of delay when compared to normal, naïve and deterministic routing. Now with better next hop selection using angle-based routing with the help of GPS, our proposed algorithm does slightly better than anycast (optimal); since it eliminates detours and focuses on direction.

\section{Conclusion}

Fast and energy efficient data transfer is of prime interest in Wireless Sensor Networks (WSN). With core limitations on sensor node resources like battery power and sensing range, such performance degradation leads to decrease in network lifetime. This is not viable for critical event monitoring. So this issue was addressed focusing on energy and delay which would have a direct impact on the QoS parameters in WSN. The proposed work 


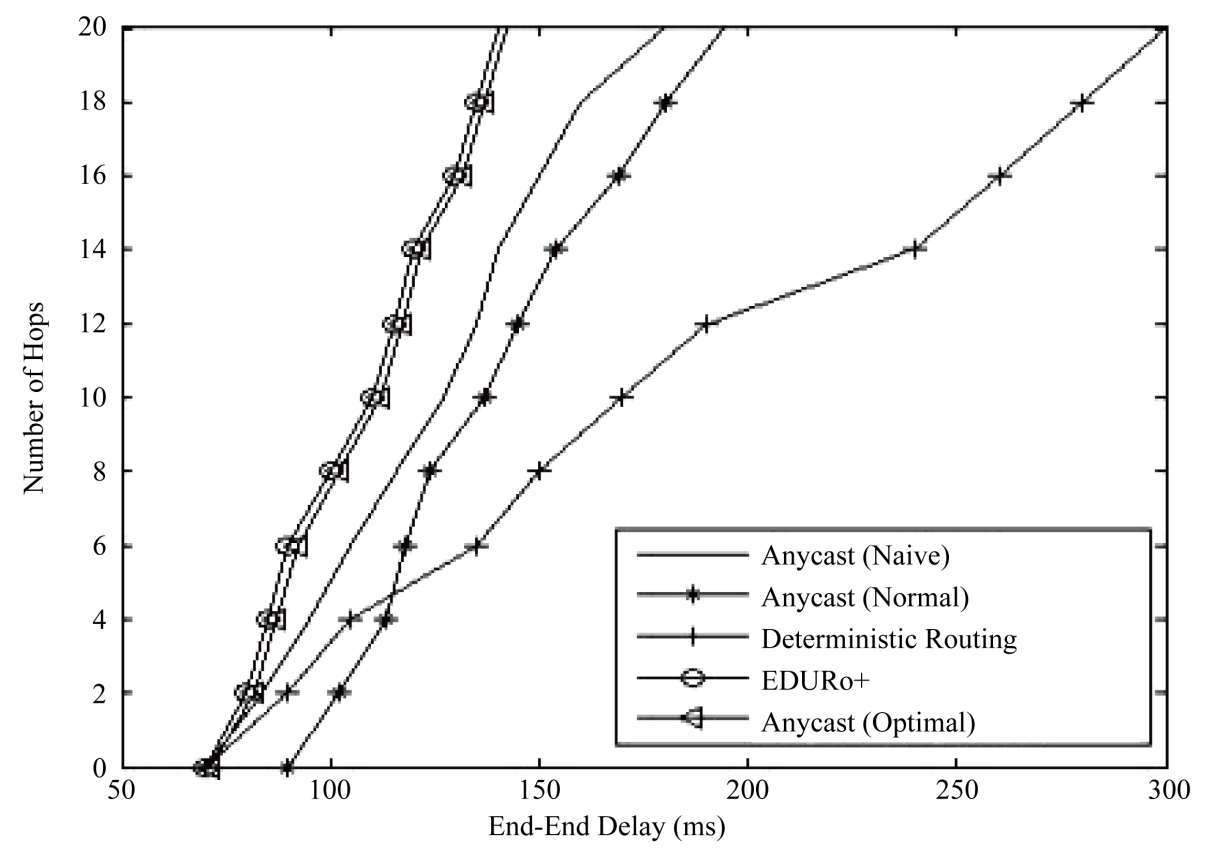

Figure 11. Performance evaluation of delay.

avoids collision between packets and provides increased energy efficiency with minimum latency and further prolonging the network lifetime. The EDURo ${ }^{+}$approach alleviates performance deficiencies in the WSN such as energy and delay by employing an angle based anycast routing methodology assisted by GPS to establish direction. It also includes a mechanism, namely prim's dual for selecting the next hop using five crucial parameters in the multi-hop packet transfer. The protocol provides several opportunities for performance enhancement by maintaining conflict free concurrent transactions. The future work requires the adoption of a routing protocol to the dynamic challenging environments like disaster management system, etc. by jointly considering the power control and to guarantee a trade-off among multi-constrained QoS in WSNs.

\section{References}

[1] Guo, P., Jiang, T., Zhang, Q. and Zhang, K. (2012) Sleep Scheduling for Critical Event Monitoring in Wireless Sensor Networks. IEEE Transactions on Parallel and Distributed Systems, 23, 345-352. http://dx.doi.org/10.1109/TPDS.2011.165

[2] Baccour, N., Koubaa, A., Motolla, L., Zuniga, M., Youssef, H., Boano, C. and Alves, M. (2011) Radio Link Quality Estimation in Wireless Sensor Networks: A Survey. ACM Transactions on Sensor Network, 8, 1-34. http://dx.doi.org/10.1145/2240116.2240123

[3] Low Rate Wireless Personal Area Networks, IEEE Standard 802.15.4/D18. http://www.iith.ac.in/ tbr/teaching/docs/802.15.4-2003.pdf

[4] Vahabi, M., Ghazvini, M.H.F., Rasid, M.F.A. and Abdullah, R.S.A.R. (2007) Trade-Off between Energy Consumption and Target Delay for Wireless Sensor Network. IEEE International Conference on Telecommunications and Malaysia International Conference on Communications, Penang, 14-17 May 2007. http://dx.doi.org/10.1109/ICTMICC.2007.4448697

[5] Su, W.L. and Lim, T.L. (2006) Cross-Layer Design and Optimization for Wireless Sensor Networks. Seventh ACIS International Conference on SNPD, 278-284.

[6] Melodia, T., Pompili, D. and Akyildiz, I.F. (2004) Optimal Local Topology Knowledge for Energy Efficient Geographical Routing in Sensor Networks. 23rd Annual Joint Conference of the IEEE Computer and Communications Societies, 3, 1705-1716. http://dx.doi.org/10.1109/infcom.2004.1354582

[7] Nath, S. and Gibbons, P.B. (2007) Communicating via Fireflies: Geographic Routing on Duty Cycled Sensors. Proceedings of IPSN, 440-449. http://dx.doi.org/10.1145/1236360.1236416

[8] Zhu, C.S., Yang, L.T. and Shu, L. (2014) Sleep Scheduling for Geographic Routing in Duty-Cycled Mobile Sensor Networks. IEEE Transactions on Industrial Electronics, 61, 6346-6355. http://dx.doi.org/10.1109/TIE.2014.2311390 
[9] Liang, L.L., Liu, X.N., Wang, Y.T., Feng, W.D. and Yang, G. (2014) SW-MAC: A Latency MAC protocol with Adaptive Sleeping for Wireless Sensor Networks. Wireless Personal Communications, 77, 1191-1211. http://dx.doi.org/10.1007/s11277-013-1561-6

[10] Polastre, J., Hill, J. and Culler, D. (2004) Versatile Low Power Media Access for Wireless Sensor Networks. Proceedings of the 2nd International Conference on Embedded Networked Sensor Systems-SenSys. http://dx.doi.org/10.1145/1031495.1031508

[11] Buettner, M., Yee, G.V., Anderson, E. and Han, R. (2006) X-MAC: A Short Preamble MAC Protocol for Duty-Cycled Wireless Sensor Networks. Proceedings of the 4th International Conference on Embedded Networked Sensor Systems, Boulder, 1-3 November 2006, 307-320. http://dx.doi.org/10.1145/1182807.1182838

[12] Moss, D. and Levis, P. (2010) BoX-MACs: Exploiting Physical and Link Layer Boundaries in Low-Power Networking. Technical Report SING-08-00, Stanford University, Stanford. http://sing.stanford.edu/pubs/sing-08-00.pdf

[13] Dutta, P., Dawson-Haggerty, S., Chen, Y., Mike Liang, C.-J. and Terzis, A. (2010) Design and Evaluation of a Versatile and Efficient Receiver-Initiated Link Layer for Low-Power Wireless. Proceedings of the 8th ACM Conference on Embedded Networked Sensor Systems, Zurich, 3-5 November 2010, 1-14.

[14] Won, M., Park, T. and Son, S.H. (2014) Asym-MAC: A MAC Protocol for Low-Power Duty-Cycled Wireless Sensor Networks with Asymmetric Links. IEEE Communications Letters, 18, 809-812. http://dx.doi.org/10.1109/LCOMM.2014.032014.132679

[15] Hung, M.C.-C., Lin, K.C.-J., Chou, C.-F. and Hsu, C.-C. (2013) EFFORT: Energy-Efficient Opportunistic Routing Technology in Wireless Sensor Networks. Wireless Communications and Mobile Computing, 13, 760-773.

[16] Wei, H., Ganguly, S., Izmailov, R. and Hass, Z.J. (2005) Interference-Aware IEEE 802.16 WiMax Mesh Networks. 5, 2005 IEEE 61st Vehicular Technology Conference, 30 May-1 June 2005, Stockholm, 3102-3106.

[17] Kwon, S. and Shroff, N.B. (2012) Energy-Efficient Unified Routing Algorithm for Multi-Hop Wireless Networks. IEEE Transactions on Wireless Communications, 11, 3890-3899.

[18] Hou, Y.T., Shi, Y. and Sherali, H.D. (2005) On Base Station Selection for Anycast Flow Routing in Energy-Constrained Wireless Sensor Networks. 2nd International Conference on Quality of Service in Heterogeneous Wired/ Wireless Networks (QSHINE’05), Lake Vista, 24 August 2005.

[19] Hou, Y.T., Shi, Y. and Sherali, H.D. (2006) Optimal Base Station Selection for Anycast Routing in Wireless Sensor Networks. IEEE Transactions on Vehicular Technology, 55, 813-821.

[20] Du, H.W., Jia, X.H. and Hu, X.D. (2006) Energy Efficient Geographic Anycast in Wireless Sensor Networks. TENCON 2006-2006 IEEE Region 10 Conference, 14-17 November 2006, 1-4. http://dx.doi.org/10.1109/tencon.2006.344162

[21] Tan, H.-X. and Chan, M.C. (2010) A²-MAC: An Adaptive, Anycast MAC Protocol for Wireless Sensor Networks. 2010 IEEE Wireless Communication and Networking Conference, Sydney, 18-21 April 2010, 1-6.

[22] Gao, D., Qian, H., Wang, Z. and Chen, J. (2011) Study and Design an Anycast Routing Protocol for Wireless Sensor Networks. Journal of Networks, 6, 1726-1733.

[23] Liu, S., Fan, K.-W. and Sinha, P. (2007) CMAC: An Energy Efficient MAC Layer Protocol Using Convergent Packet Forwarding for Wireless Sensor Networks. 2007 4th Annual IEEE Communications Society Conference on Sensor, Mesh and Ad Hoc Communications and Networks, San Diego, 18-21 June 2007, 11-20. http://dx.doi.org/10.1109/sahcn.2007.4292813

[24] Kim, J., Lin, X.J., Shroff, N.B. andSinha, P. (2010) Minimizing Delay and Maximizing Lifetime for Wireless Sensor Networks With Anycast. IEEE/ACM Transactions on Networking, 18, 515-528. http://dx.doi.org/10.1109/TNET.2009.2032294

[25] Wang, X.M., Wang, J.P., Lu, K.J. and Xu, Y.L. (2013) GKAR: A Novel Geographic K-Anycast Routing for Wireless Sensor Networks. IEEE Transactions on Parallel and Distributed Systems, 24, 916-925.

[26] Mao, X., Tang, S., Xu, X., Li, X. and Ma, H. (2011) Energy Efficient Opportunistic Routing in Wireless Sensor Networks. IEEE Transactions on Parallel and Distributed Systems, 22, 1934-1942. http://dx.doi.org/10.1109/TPDS.2011.70

[27] Hsu, C.-C., Kuo, M.-S., Wang, S.-C. and Chou, C.-F. (2014) Joint Design of Asynchronous Sleep-Wake Scheduling and Opportunistic Routing in Wireless Sensor Networks. IEEE Transactions on Computers, 63, 1840-1846.

[28] Park, J. and Sahni, S. (2006) An Online Heuristic for Maximum Lifetime Routing in Wireless Sensor Networks. IEEE Transactions on Computers, 55, 1048-1056. http://dx.doi.org/10.1109/TC.2006.116

[29] Luo, J., Hu, J.Y., Wu, D. and Li, R.F. (2015) Opportunistic Routing Algorithm for Relay Node Selection in Wireless Sensor Networks. IEEE Transactions on Industrial Informatics, 11, 112-121. http://dx.doi.org/10.1109/TII.2014.2374071 